\title{
Correction to: Protective Effect on Mortality of Active Commuting to Work: A Systematic Review and Meta-analysis
}

\author{
Frédéric Dutheil ${ }^{1,2}$ (1) Séverine Pélangeon ${ }^{3} \cdot$ Martine Duclos $^{4} \cdot$ Philippe Vorilhon $^{5} \cdot$ Martial Mermillod $^{6,7}$. \\ Julien S. Baker ${ }^{8} \cdot$ Bruno Pereira $^{9} \cdot$ Valentin Navel $^{10}{ }^{10}$
}

Published online: 26 October 2020

(c) Springer Nature Switzerland AG 2020

\section{Correction to: Sports Medicine https://doi.org/10.1007/s40279-020-01354-0}

Within the abstract, the following sentence: "Using the fully adjusted estimates of risks, active commuting decreased allcause mortality by $9 \%$ (95\% confidence intervals $3-9 \%$ ), and cardiovascular mortality by $15 \%(3-27 \%)(\mathrm{p}<0.001)$." should be written as "Using the fully adjusted estimates of risks, active commuting decreased all-cause mortality by $9 \%$ (95\% confidence intervals 3-15\%), and cardiovascular mortality by $15 \%(3-27 \%)(\mathrm{p}<0.001) . "$

The original article has been updated.

The original article can be found online at https://doi.org/10.1007/ s40279-020-01354-0.

Frédéric Dutheil

frederic.dutheil@acu.edu.au

1 CNRS, LaPSCo, Physiological and Psychosocial Stress, CHU Clermont-Ferrand, University Hospital of Clermont-Ferrand, Preventive and Occupational Medicine, Université Clermont Auvergne, Witty Fit, 63000 Clermont-Ferrand, France

2 Faculty of Health, School of Exercise Science, Melbourne, Australia2 General Medicine, AMUAC, Australian Catholic University, 63000 Clermont-Ferrand, France

3 General Medicine, AMUAC, 63000 Clermont-Ferrand, France

4 INRA, UMR 1019, CRNH-Auvergne, University Hospital of Clermont-Ferrand, CHU Clermont-Ferrand, Sport Medicine and Functional Explorations, Université Clermont Auvergne, 63000 Clermont-Ferrand, France
5 Université Clermont Auvergne, UPU ACCePPT, 63000 Clermont-Ferrand, France

6 Université Grenoble Alpes, Université Savoie Mont Blanc, CNRS, LPNC, 38000 Grenoble, France

7 Institut Universitaire de France, Paris, France

8 Centre for Health and Exercise Science Research, Department of Sport, Physical Education and Health, Hong Kong Baptist University, Kowloon Tong, Hong Kong

9 CHU Clermont-Ferrand, Clinical Research and Innovation Direction, University Hospital of Clermont Ferrand, 63000 Clermont-Ferrand, France

10 CNRS, INSERM, GReD, Translational Approach To Epithelial Injury and Repair, CHU Clermont-Ferrand, University Hospital of Clermont-Ferrand, Ophthalmology, Université Clermont Auvergne, 63000 Clermont-Ferrand, France 